\title{
TeV-scale neutrino mass generation, minimal inelastic dark matter, and high scale leptogenesis
}

\author{
Pei-Hong $\mathrm{Gu}^{1, *}$ and Hong-Jian $\mathrm{He}^{1,2,3,4, \dagger}$ \\ ${ }^{1}$ School of Physics and Astronomy, Shanghai Jiao Tong University, Shanghai 200240, China \\ ${ }^{2}$ T. D. Lee Institute, Shanghai 200240, China \\ ${ }^{3}$ Institute of Modern Physics, Tsinghua University, Beijing 100084, China \\ ${ }^{4}$ Center for High Energy Physics, Peking University, Beijing 100871, China
}

(Received 21 September 2018; published 18 January 2019)

\begin{abstract}
The seesaw and leptogenesis commonly depend on the masses of the same particles and thus are both realized at the same scale. In this work, we demonstrate a new possibility to realize a $\mathrm{TeV}$-scale neutrino seesaw and a natural high-scale leptogenesis. We extend the standard model by two gauge-singlet scalars, a vectorlike isodoublet fermion and one isotriplet Higgs scalar. Our model respects a softly broken lepton number and an exactly conserved $\mathbb{Z}_{2}$ discrete symmetry. It can achieve three things altogether: (i) realizing a testable type-II seesaw at the $\mathrm{TeV}$ scale with two nonzero neutrino mass eigenvalues, (ii) providing minimal inelastic dark matter from the new fermion doublets, and (iii) accommodating a thermal or nonthermal leptogenesis through the singlet scalar decays. We further analyze the current experimental constraints on our model and discuss the implications for the dark matter direct detections and the LHC searches.
\end{abstract}

DOI: 10.1103/PhysRevD.99.015025

\section{INTRODUCTION}

The seesaw [1,2] extensions of the standard model (SM) naturally explain the tiny neutrino masses [3], while accommodating a leptogenesis [4-6] mechanism to generate the observed cosmic baryon asymmetry [3]. In the conventional seesaw-leptogenesis scenarios, the scales of generating neutrino masses and baryon asymmetry are tied together and determined by the masses of the same particles. The leptogenesis cannot be realized at the $\mathrm{TeV}$ scale unless it invokes a large fine-tuning to resonantly enhance the required $C P$ asymmetry. This means that a natural leptogenesis is achieved at high scale, and the conventional scenarios link the seesaw to the same leptogenesis scale, which prevent the realization of testable seesaw at the $\mathrm{TeV}$ scale.

The strong evidence for nonbaryonic dark matter (DM) poses another great challenge to modern particle physics and cosmology [3]. There have been interesting ideas explaining the DM puzzle. For instance, the minimal DM models [7-9] can give testable predictions for DM

\footnotetext{
peihong.gu@sjtu.edu.cn

"hjhe@tsinghua.edu.cn
}

Published by the American Physical Society under the terms of the Creative Commons Attribution 4.0 International license. Further distribution of this work must maintain attribution to the author(s) and the published article's title, journal citation, and DOI. Funded by SCOAP. properties including the DM mass and the DM-nucleon scattering. However, for models with the new weak multiplet of nonzero hypercharge, its neutral DM component will have gauge interactions with $Z^{0}$ and thus is excluded by the direct DM searches [7]. This calls for viable extensions. Besides, the DM particle may also play an important role in the generation of neutrino masses [10-14] and the realization of baryon asymmetry [12].

In this work, we propose an attractive possibility that new physics for generating a testable $\mathrm{TeV}$-scale seesaw can accommodate a thermal or inflationary baryogenesis at a very high scale. At the same time, we provide a viable minimal inelastic DM candidate at the $\mathrm{TeV}$ scale. In our construction, we will construct a realistic model including two gauge-singlet scalars, a vectorlike isodoublet fermion and one isotriplet Higgs scalar besides the SM fields. Our model has a softly broken lepton number and an exactly conserved $\mathbb{Z}_{2}$ discrete symmetry, so it differs from other models [15] with a spontaneous breaking lepton or baryon number. Under such a softly broken lepton number and the exact $\mathbb{Z}_{2}$ symmetry, our model can achieve three things altogether: (i) realizing a testable type-II seesaw at $\mathrm{TeV}$ scale with two nonzero neutrino mass eigenvalues; (ii) providing a minimal inelastic dark matter from the new fermion doublets, with the mass-splitting induced by interactions related to the neutrino mass generations; and (iii) accommodating a thermal or inflationary leptogenesis at a high scale through the scalar-singlet decays. Although the leptogenesis scale is high, realizing the DM relic 
density in our scenario requires the DM mass to be about 1.2 TeV. As we will show, the present minimal inelastic DM is a stable Majorana fermion and depends on two new parameters: the DM mass and the mass difference between the DM and another particle. (This differs from the previous minimal DM model [7-9] in which the DM is either a scalar or Dirac fermion, and its tree-level mass is the only new physics parameter.) The predicted Higgs triplet and DM fermion of our model can be searched at the LHC and future high-energy $p p$ colliders. The same DM particle can be probed by the direct and indirect DM detection experiments [16].

This paper is organized as follows. In Sec. II, we present the model setup. Then, we study the minimal inelastic DM in Sec. III and the radiative type-II neutrino seesaw in Sec. IV. The realization of high scale leptogenesis is presented Sec. V. Finally, we conclude in Sec. VI.

\section{MODEL CONSTRUCTION}

In this section, we present the model setup and discuss the involved (un)broken symmetries. For the current model, we introduce two gauge-singlet scalars,

$$
\sigma_{j}(1,1,0)=\frac{1}{\sqrt{2}}\left(\sigma_{j R}+i \sigma_{j I}\right), \quad(j=1,2) ;
$$

a vectorlike isodoublet fermion (with left-handed component $\psi_{L}$ and right-handed component $\psi_{L}^{\prime}{ }^{c}$ ),

$\psi_{L}\left(1,2,+\frac{1}{2}\right)=\left(\begin{array}{c}\xi_{L}^{+} \\ \chi_{L}\end{array}\right), \quad \psi_{L}^{\prime}\left(1,2,-\frac{1}{2}\right)=\left(\begin{array}{c}\chi_{L}^{\prime} \\ \xi_{L}^{\prime}\end{array}\right) ;$

and an isotriplet scalar,

$$
\Delta(1,3,-1)=\left(\begin{array}{cc}
\frac{1}{\sqrt{2}} \delta^{-} & \delta^{0} \\
\delta^{--} & -\frac{1}{\sqrt{2}} \delta^{-}
\end{array}\right)
$$

On the left-hand side of each equation above, the numbers in the parentheses describe the representations (or quantum numbers) of the corresponding field under the SM gauge group $S U(3)_{c} \otimes S U(2)_{L} \otimes U(1)_{Y}$. This model also respects a softly broken lepton number and an exactly conserved $\mathbb{Z}_{2}$ discrete symmetry. By definition, only the scalar singlets $\sigma_{j}(j=1,2)$ carry a lepton number -1 , which is opposite to the SM leptons. Under the $\mathbb{Z}_{2}$ discrete symmetry, the SM fields and the scalar triplet $\Delta$ are $\mathbb{Z}_{2}$ even, while the scalar singlets $\sigma_{j}$ and the fermion doublets $\left(\psi_{L}, \psi_{L}^{\prime}\right)$ are $\mathbb{Z}_{2}$ odd. Thus, we have the following $\mathbb{Z}_{2}$ transformations:

$$
\begin{aligned}
(\mathrm{SM}, \Delta) & \stackrel{\mathbb{Z}_{2}}{\rightarrow}(\mathrm{SM}, \Delta), \\
\left(\sigma_{j}, \psi_{L}, \psi_{L}^{\prime}\right) & \stackrel{\mathbb{Z}_{2}}{\rightarrow}-\left(\sigma_{j}, \psi_{L}, \psi_{L}^{\prime}\right) .
\end{aligned}
$$

Since the $\mathbb{Z}_{2}$ discrete symmetry is exactly conserved, the scalar singlets $\sigma_{j}$ will not acquire any nonzero vacuum expectation value (VEV).

For the current analysis, we write down the relevant Lagrangian terms

$$
\begin{aligned}
\mathcal{L} \supset & -\sigma^{\dagger} M_{\sigma}^{2} \sigma-\frac{1}{2} \sigma^{T} \tilde{M}_{\sigma}^{2} \sigma-M_{\Delta}^{2} \operatorname{Tr}\left(\Delta^{\dagger} \Delta\right)+\mu_{\Delta \phi} \phi^{T} \dot{\mathrm{i}} \tau_{2} \Delta \phi \\
& -M_{\psi} \overline{\psi_{L}^{c}} \dot{\mathrm{i}} \tau_{2} \psi_{L}^{\prime}+\frac{1}{2} f \overline{\psi_{L}^{c}} \dot{\mathrm{i}} \tau_{2} \Delta \psi_{L}-\frac{1}{2} f^{\prime} \overline{\psi_{L}^{c}} \dot{\mathrm{i}} \tau_{2} \Delta^{\dagger} \psi_{L}^{\prime} \\
& -y_{\alpha j} \overline{L_{L \alpha}^{c}} \mathrm{i} \tau_{2} \psi_{L} \sigma_{j}+\text { H.c. },
\end{aligned}
$$

where $\tau_{2}$ is the second Pauli matrix and $\sigma=\left(\sigma_{1}, \sigma_{2}\right)^{T}$ denotes the singlet scalars. In Eq. (5), the fields $\phi, L_{L}$, and $e_{R}$ denote the Higgs doublet, the left-handed lepton doublet, and the right-handed lepton in the SM, respectively. Thus, the Higgs doublet $\phi$ and the left-handed lepton doublet $L_{L}$ take the following form:

$\phi\left(1,2,+\frac{1}{2}\right)=\left(\begin{array}{c}\phi^{+} \\ \phi^{0}\end{array}\right), \quad L_{L}\left(1,2,-\frac{1}{2}\right)=\left(\begin{array}{c}\nu_{L} \\ e_{L}\end{array}\right)$.

It is clear that in Eq. (5) the $\tilde{M}_{\sigma}^{2}$ mass term is the unique source of the lepton-number violation, since by construction only the SM leptons and the scalar singlets $\sigma_{j}$ carry lepton numbers. We note that requiring the softly broken lepton number and the exactly conserved $\mathbb{Z}_{2}$ discrete symmetry has forbidden the following gauge-invariant terms,

$$
\begin{aligned}
\mathcal{L} \not & -M^{\prime} \overline{\psi_{L}^{c}} \dot{\mathrm{i}} \tau_{2} L_{L}-\frac{1}{2} f^{\prime \prime} \overline{\psi^{\prime c}}{ }_{L}^{\mathrm{i}} \tau_{2} \Delta^{\dagger} L_{L}-\frac{1}{2} f^{\prime \prime \prime} \overline{L_{L}^{c}} \dot{\mathrm{i}} \tau_{2} \Delta^{\dagger} L_{L} \\
& -f_{j}^{\prime \prime \prime} \bar{L}_{L} \psi_{L}^{\prime} \sigma_{j}-y^{\prime} \overline{\psi_{L}^{\prime}} \phi e_{R}+\text { H.c. },
\end{aligned}
$$

with $e_{R}(1,1,-1)$ being the right-handed leptons.

For simplicity, we consider the real mass parameters in the present analysis,

$$
\mu_{\Delta \phi}=\mu_{\Delta \phi}^{*}, \quad M_{\psi}=M_{\psi}^{*} .
$$

Then, we choose the singlet mass matrix $M_{\sigma}^{2}$ to be diagonal, and for simplicity of the analysis, we further assume $\tilde{M}_{\sigma}^{2}$ to be diagonal as well,

$M_{\sigma}^{2}=\operatorname{diag}\left\{M_{\sigma_{1}}^{2}, M_{\sigma_{2}}^{2}\right\}, \quad \tilde{M}_{\sigma}^{2}=\operatorname{diag}\left\{\tilde{M}_{\sigma_{1}}^{2}, \tilde{M}_{\sigma_{2}}^{2}\right\}$.

Accordingly, we can deduce the mass eigenvalues of the real and imaginary components of the two singlet scalars $\sigma_{1,2}$ as follows:

$$
\begin{aligned}
& \mathcal{L}_{\text {mass }}^{\sigma}=-\frac{1}{2} M_{\sigma_{j R}}^{2} \sigma_{j R}^{2}-\frac{1}{2} M_{\sigma_{j l}}^{2} \sigma_{i I}^{2}, \\
& M_{\sigma_{j R}}^{2}=M_{\sigma_{j}}^{2}+\tilde{M}_{\sigma_{j}}^{2}, \quad M_{\sigma_{j l}}^{2}=M_{\sigma_{j}}^{2}-\tilde{M}_{\sigma_{j}}^{2} .
\end{aligned}
$$


For simplicity of demonstration, we also set both Yukawa couplings $f$ and $f^{\prime}$ be real,

$$
f=f^{*}, \quad f^{\prime}=f^{*},
$$

although one of them is allowed to be complex.

\section{MINIMAL INELASTIC DARK MATTER}

In this section, we further analyze the model predictions and the experimental constraints. In particular, we shall identify a stable Majorana fermion as a viable DM particle of mass $\sim 1.2 \mathrm{TeV}$. We show that the present inelastic DM model depends on two new parameters: the DM mass and the mass difference between the DM and another particle. This differs from the previous minimal DM model [7-9] in which the DM is either a scalar or Dirac fermion, and its tree-level mass is the only new physics parameter.

In the present model, the SM Higgs doublet $\phi$ will develop a VEV for spontaneous electroweak symmetry breaking at the weak scale. The scalar triplet $\Delta$ has a positive mass term and will acquire an induced VEV, due to its cubic interaction with the SM Higgs doublet $\phi$ via the $\mu_{\Delta \phi}$ term in Eq. (5). So, we will refer the scalar triplet $\Delta$ as a Higgs triplet. The Higgs scalars $\phi$ and $\Delta$ have 10 degrees of freedom (d.o.f.) in total, including four real neutral scalars, two singly charged scalars, and one doubly charged scalar,

$$
\begin{aligned}
& \phi=\left(\begin{array}{c}
\phi^{+} \\
\frac{1}{\sqrt{2}}\left(v_{\phi}+h_{\phi}+i \phi_{I}^{0}\right)
\end{array}\right), \\
& \Delta=\left(\begin{array}{cc}
\frac{1}{\sqrt{2}} \delta^{-} & \frac{1}{\sqrt{2}}\left(v_{\Delta}+h_{\Delta}+i \delta_{I}^{0}\right) \\
\delta^{--} & -\frac{1}{\sqrt{2}} \delta^{-}
\end{array}\right),
\end{aligned}
$$

with the VEVs $v_{\phi}$ and $v_{\Delta}$ from their neutral components. Among the d.o.f. in the Higgs scalars $\phi$ and $\Delta$, one neutral massless eigenstate from $\phi_{I}^{0}$ and $\delta_{I}^{0}$ as well as one pair of charged massless eigenstates from $\phi^{ \pm}$and $\delta^{ \pm}$will be absorbed by the longitudinal components of the weak gauge bosons $Z^{0}$ and $W^{ \pm}$. The VEVs $v_{\phi}$ and $v_{\Delta}$ should be subject to the precision constraints [3],

$$
\begin{aligned}
& v \equiv \sqrt{v_{\phi}^{2}+2 v_{\Delta}^{2}} \simeq 246 \mathrm{GeV}, \\
& \rho=\frac{v_{\phi}^{2}+2 v_{\Delta}^{2}}{v_{\phi}^{2}+4 v_{\Delta}^{2}}=1.00040 \pm 0.00024
\end{aligned}
$$

With the $3 \sigma$ lower limit $\rho \geq 0.99968$, we deduce

$$
\left|v_{\Delta}\right|=\sqrt{\frac{1-\rho}{2 \rho}} v \leq 3.1 \mathrm{GeV}
$$

From the relevant scalar potential terms in Eq. (5), we derive $v_{\Delta}$ as

$$
v_{\Delta} \simeq \frac{\mu_{\Delta \phi} v_{\phi}^{2}}{\sqrt{2} M_{\Delta}^{2}},
$$

for $M_{\Delta} \gg \mu_{\Delta \phi}$, or $M_{\Delta} \gg v_{\phi}$. We see that a small triplet VEV $v_{\Delta}$ is naturally generated in the present model, due to the seesaw-type suppression in the above formula (15).

According to the Yukawa interactions in Eq. (5), the small VEV $v_{\Delta}$ of Higgs triplet will also contribute to Majorana masses of the neutral fermions $\chi_{L}$ and $\chi_{L}^{\prime}$. Thus, we derive the following mass terms for charged fermions $\left(\xi_{L}^{ \pm}, \xi_{L}^{\prime \pm}\right)$ and neutral fermions $\left(\chi_{L}, \chi_{L}^{\prime}\right)$ :

$$
\begin{aligned}
\mathcal{L}_{\text {mass }}^{\xi \chi}= & -M_{\psi} \overline{\xi_{L}^{+c}} \xi_{L}^{\prime}+M_{\psi} \overline{\chi_{L}^{c}} \chi_{L}^{\prime} \\
& -\frac{v_{\Delta}}{2 \sqrt{2}}\left(f \overline{\chi_{L}^{c}} \chi_{L}+f^{\prime} \overline{\chi_{L}^{\prime c}} \chi_{L}^{\prime}\right)+\text { H.c. }
\end{aligned}
$$

For convenience, we can express the Dirac spinors in terms of the left-handed Weyl spinors in the $\left(\frac{1}{2}, 0\right)$ representation of the Lorentz group,

$$
\begin{array}{ll}
\chi_{L}=\left(\begin{array}{c}
\chi \\
0
\end{array}\right), & \chi_{L}^{\prime}=\left(\begin{array}{c}
\chi^{\prime} \\
0
\end{array}\right), \\
\xi_{L}^{ \pm}=\left(\begin{array}{c}
\xi^{ \pm} \\
0
\end{array}\right), & \xi_{L}^{ \pm}=\left(\begin{array}{c}
\xi^{ \pm \pm} \\
0
\end{array}\right) .
\end{array}
$$

Thus, we can rewrite the mass terms (16) as

$$
\begin{aligned}
\mathcal{L}_{\text {mass }}^{\xi \chi}= & M_{\psi} \xi^{+T} \epsilon \xi^{\prime-}-M_{\psi} \chi^{T} \epsilon \chi^{\prime} \\
& +\frac{v_{\Delta}}{2 \sqrt{2}}\left(f \chi^{T} \epsilon \chi+f^{\prime} \chi^{\prime T} \epsilon \chi^{\prime}\right)+\text { H.c. },
\end{aligned}
$$

where $\epsilon=i \tau_{2}$ is antisymmetric. We see that the two charged Weyl spinors $\xi^{ \pm}$and $\xi^{\prime \pm}$ form a Dirac mass term with mass $M_{\xi}=M_{\psi}$. Defining the charged Dirac spinor,

$$
\tilde{\xi}^{ \pm}=\left(\begin{array}{c}
\xi^{ \pm} \\
\epsilon \xi^{\xi^{\mp *}}
\end{array}\right)=\xi_{L}^{ \pm}+\left(\xi_{L}^{\prime \mp}\right)^{c}
$$

we can express the Dirac mass term $M_{\psi} \xi^{+T} \epsilon \xi^{\prime-}+$ H.c. in the conventional 4-component form $M_{\psi} \bar{\xi}^{+} \tilde{\xi}^{+}$. From Eq. (18), the neutral fermions $\hat{\chi} \equiv\left(\chi, \chi^{\prime}\right)^{T}$ have the Majorana mass term $-\frac{1}{2} \hat{\chi}^{T} \mathbb{M}_{\chi} \hat{\chi}$ with mass matrix,

$$
\mathbb{M}_{\chi}=\left(\begin{array}{cc}
-f \bar{v}_{\Delta} & M_{\psi} \\
M_{\psi} & -f^{\prime} \bar{v}_{\Delta}
\end{array}\right),
$$

where $\bar{v}_{\Delta}=v_{\Delta} / \sqrt{2}$. Then, we can diagonalize the symmetric mass matrix $\mathbb{M}_{\chi}$ and derive the mass eigenvalues 


$$
\begin{aligned}
& M_{\chi_{1}}=\sqrt{M_{\psi}^{2}+\frac{v_{\Delta}^{2}}{8}\left(f-f^{\prime}\right)^{2}}-\frac{f+f^{\prime}}{2 \sqrt{2}} v_{\Delta}, \\
& M_{\chi_{2}}=\sqrt{M_{\psi}^{2}+\frac{v_{\Delta}^{2}}{8}\left(f-f^{\prime}\right)^{2}}+\frac{f+f^{\prime}}{2 \sqrt{2}} v_{\Delta},
\end{aligned}
$$

with $M_{\chi_{1}}<M_{\chi_{2}}$ for $f+f^{\prime}>0$. For the case $M_{\psi} \gg$ $\left|f \pm f^{\prime}\right| v_{\Delta}=\mathcal{O}(\mathrm{GeV})$, we see that the mass eigenvalues $M_{\chi_{1}}$ and $M_{\chi_{2}}$ are quite degenerate: $M_{\chi_{1}} \approx M_{\chi_{2}} \approx M_{\psi}$. For diagonalizing the mass matrix (20), we rotate the fields $\left(\chi_{1}, \chi_{2}\right)$ into their mass eigenstates $\left(\tilde{\chi}_{1}, \tilde{\chi}_{2}\right)$ by the unitary rotation $\left(\chi, \chi^{\prime}\right)^{T}=U\left(\chi_{1}, \text { i } \chi_{2}\right)^{T}$ with

$$
U=\left(\begin{array}{rr}
\cos \theta & \sin \theta \\
-\sin \theta & \cos \theta
\end{array}\right)
$$

Thus, we determine the rotation angle $\theta$ as follows:

$$
\tan 2 \theta=\frac{2 \sqrt{2} M_{\psi}}{\left(f-f^{\prime}\right) v_{\Delta}}
$$

For the case $M_{\psi} \gg\left|f-f^{\prime}\right| v_{\Delta}=\mathcal{O}(\mathrm{GeV})$, we have the rotation angle $\theta \simeq \frac{\pi}{4}$.

With these, we can rewrite the mass term (18) in the diagonalized form:

$\mathcal{L}_{\text {mass }}^{\xi \chi}=M_{\psi} \bar{\xi}^{+} \tilde{\xi}^{+}-\frac{1}{2}\left(M_{\chi_{1}} \chi_{1}^{T} \epsilon \chi_{1}+M_{\chi_{2}} \chi_{2}^{T} \epsilon \chi_{2}\right)+$ H.c.

Then, we can derive gauge interactions of the charged Dirac fermions $\tilde{\xi}^{ \pm}$and the neutral Majorana fermions $\chi_{1,2}$. In the usual 4-component notations, we denote $\chi_{L j}=\left(\chi_{j}, 0\right)^{T}$ $(j=1,2)$. Thus, we can express the gauge interactions of $\tilde{\xi}^{ \pm}$and $\chi_{L 1,2}$ as

$$
\begin{aligned}
\mathcal{L}_{\mathrm{G}}^{\xi \chi}= & e \overline{\tilde{\xi}^{+}} \gamma^{\mu} \tilde{\xi}^{+} A_{\mu}+\frac{g \cos 2 \theta_{W}}{2 \cos \theta_{W}} \overline{\tilde{\xi}^{+}} \gamma^{\mu} \tilde{\xi}^{+} Z_{\mu} \\
& +\frac{g}{2 \cos \theta_{W}} \overline{\chi_{L 1}} \gamma^{\mu} \chi_{L 2} Z_{\mu}-\frac{g}{2} \overline{\chi_{L 1}} \gamma^{\mu} \tilde{\xi}^{+} W_{\mu}^{-} \\
& -\frac{g}{2} \overline{\chi_{L 2}} \gamma^{\mu} \tilde{\xi}^{+} W_{\mu}^{-}+\text {H.c. }
\end{aligned}
$$

where we have set $\theta \simeq \frac{\pi}{4}$, which holds well for $M_{\psi} \gg\left(f+f^{\prime}\right) v_{\Delta}$.

As Eq. (14) restricts the Higgs triplet VEV $v_{\Delta}$ within a few $\mathrm{GeV}$, we find that the mass splitting between the Majorana fermions $\left(\chi_{1}, \chi_{2}\right)$ is constrained as

$$
\begin{aligned}
\Delta M_{\chi} & =M_{\chi_{2}}-M_{\chi_{1}}=\frac{1}{\sqrt{2}}\left(f+f^{\prime}\right) v_{\Delta} \\
& =17.1 \mathrm{GeV}\left(\frac{f+f^{\prime}}{2 \sqrt{4 \pi}}\right)\left(\frac{v_{\Delta}}{3.1 \mathrm{GeV}}\right) \lesssim 17.1 \mathrm{GeV},
\end{aligned}
$$

which is much smaller than the $\chi_{1,2}$ masses themselves. This means that the Majorana fermions $\chi_{1,2}$ are quasidegenerate and thus are pseudo-Dirac fermions.

In the next section, we shall show that the Higgs triplet $\mathrm{VEV} v_{\Delta}$ is also responsible for the neutrino mass generations through a radiative type-II seesaw at $\mathrm{TeV}$ scale. Such a TeV-scale Higgs triplet can be tested at the LHC. The LHC could further probe the structure of the neutrino mass matrix if the VEV of this Higgs triplet is not bigger than $10^{-4} \mathrm{GeV}$ [17]. In this case, the mass splitting (26) should receive an upper bound,

$$
\Delta M_{\chi} \lesssim 550 \mathrm{keV}
$$

for $|f|,\left|f^{\prime}\right|<\sqrt{4 \pi}$ and $v_{\Delta} \lesssim 10^{-4} \mathrm{GeV}$. On the other hand, even if the Majorana fermions $\chi_{1,2}$ are degenerate and thus compose a Dirac fermion $\chi=\chi_{L}+\chi_{L}^{\prime c}$, the radiative corrections from the electroweak gauge interactions will induce a mass splitting between the charged fermions $\tilde{\xi}^{ \pm}$ and the neutral fermion $\chi$,

$$
\Delta M=M_{\tilde{\xi}^{ \pm}}-M_{\chi}=\frac{g^{2} \sin ^{2} \theta_{W}}{16 \pi^{2}} M_{\psi} F\left(\frac{m_{Z}}{M_{\psi}}\right)
$$

where $\theta_{W}$ denotes the weak mixing angle and $m_{Z}$ is the mass of gauge boson $Z^{0}$. The function $F$ is defined as

$$
F(r)= \begin{cases}r^{4} \ln r-r^{2}-r\left(r^{2}-4\right)^{\frac{1}{2}}\left(r^{2}+2\right) \ln \frac{r+\sqrt{r^{2}-4}}{2}, & (\text { for } r \geq 2) \\ r^{4} \ln r-r^{2}+r\left(4-r^{2}\right)^{\frac{1}{2}}\left(r^{2}+2\right) \arctan \frac{\sqrt{4-r^{2}}}{r}, & (\text { for } r \leq 2) .\end{cases}
$$

For $M_{\psi} \gg m_{Z}$, the radiative mass splitting $\Delta M$ is well approximated as

$$
\Delta M \simeq \frac{1}{2} \alpha_{\mathrm{em}} m_{Z} \simeq 356 \mathrm{MeV}
$$

where $\alpha_{\mathrm{em}}=e^{2} / 4 \pi \simeq 1 / 128$ is the fine structure constant at the scale $\mu=m_{Z}$. If the tree-level mass splitting (26) is smaller than the radiative mass splitting (30), such as the choice (27), the charged fermions $\tilde{\xi}^{ \pm}$can decay into the neutral fermion $\chi$ with a virtual $W^{ \pm}$boson. Subsequently, 
the heavier Majorana fermion $\chi_{2}$ can decay into the lighter Majorana fermion $\chi_{1}$ with a virtual $Z$ boson. Alternatively, if the mass splitting (26) is larger than the mass splitting (30), the heavier $\chi_{2}$ can simultaneously decay into $\tilde{\xi}^{ \pm}$and $W^{*}$, and then $\tilde{\xi}^{ \pm}$will decay into the lighter $\chi_{1}$ plus $W^{*}$. In either case, all of the decay chains can be completed before the big bang nucleosynthesis (BBN) epoch. For instance, we may consider the loop-induced process $\chi_{2} \rightarrow \chi_{1} \gamma$, which always exists [for any mass difference in (26) or (28)] and the decay rate of which may be slower than (or comparable to) the tree-level processes mentioned above. If this loop-induced decay process is much faster than the $\mathrm{BBN}$ epoch, then the above analysis is fine. We note that the $\chi_{1}-\chi_{2}-\gamma$ vertex can arise from the dimension- 5 effective operator,

$$
\mathcal{L} \supset-\frac{i}{\Lambda} \bar{\chi}_{1} \sigma^{\mu \nu} \chi_{2} A_{\mu \nu}, \quad \text { with } \quad \frac{1}{\Lambda} \sim \frac{\left(\frac{g}{2}\right)^{2} e}{16 \pi^{2} M_{\chi}} .
$$

Thus, we can estimate the decay width,

$$
\begin{aligned}
\Gamma\left(\chi_{2} \rightarrow \chi_{1} \gamma\right) & \sim \frac{\alpha^{3}}{\sin ^{4} \theta_{W}} \frac{\left(\Delta M_{\chi}\right)^{3}}{M_{\chi}^{2}} \\
& \simeq 8.5 \times 10^{-22} \mathrm{GeV}\left(\frac{\Delta M_{\chi}}{550 \mathrm{keV}}\right)^{3}\left(\frac{M_{\chi}}{1.2 \mathrm{TeV}}\right)^{-2} \\
& \left.\gg H(T)\right|_{T=T_{\mathrm{BBN}}} \\
& \simeq 4.5 \times 10^{-25} \mathrm{GeV}\left(\frac{T_{\mathrm{BBN}}}{1 \mathrm{MeV}}\right)^{2}
\end{aligned}
$$

This shows that the typical decay rate of $\chi_{2} \rightarrow \chi_{1} \gamma$ can be much faster than the BBN epoch for the mass difference $\Delta M_{\chi} \sim 550 \mathrm{keV}$ or larger. In Eq. (32), the Hubble constant $H(T)$ is given by

$$
H(T)=\left(\frac{8 \pi^{3} g_{*}}{90}\right)^{\frac{1}{2}} \frac{T^{2}}{M_{\mathrm{Pl}}},
$$

with $M_{\mathrm{Pl}} \simeq 1.22 \times 10^{19} \mathrm{GeV}$ being the Planck mass and $g_{*}=10.75$ denoting the relativistic d.o.f. during the BBN epoch.

The lighter Majorana fermion $\chi_{1}$ will remain stable and leave a relic density in the present Universe. Equations (26) and (30) show that the charged fermions $\tilde{\xi}^{ \pm}$and neutral fermions $\chi_{1,2}$ can be fairly quasidegenerate. Thus, for computing the relic density, we should take into account not only the annihilations of the lightest $\chi_{1}$ but also the annihilations and coannihilations involving the heavier $\tilde{\xi}^{ \pm}$ and $\chi_{2}$. Such annihilation and coannihilation processes can be induced by either gauge interactions or Yukawa interactions. As we will explain after Eq. (34), our model can realize the case in which the gauge interactions dominate the annihilation and coannihilation processes, while the processes from Yukawa interactions can be negligible.
The processes induced by gauge interactions can contain in their final states the electroweak gauge bosons, the Higgs bosons, and the fermions. Thus, the corresponding effective cross section is a sum of the leading contributions with the final states of gauge bosons $(\mathrm{G})$, Higgs bosons $(\mathrm{H})$, and fermions $(\mathrm{F})$,

$$
\begin{aligned}
\langle\sigma v\rangle & \simeq\langle\sigma v\rangle_{\mathrm{G}}+\langle\sigma v\rangle_{\mathrm{H}}+\langle\sigma v\rangle_{\mathrm{F}} \\
& \simeq \frac{87 \alpha_{2}^{2}+24 \alpha_{2} \alpha_{Y}+45 \alpha_{Y}^{2}}{64 \pi^{-1} M_{\chi_{1}}^{2}},
\end{aligned}
$$

where $\left(\alpha_{2}, \alpha_{Y}\right)=\frac{1}{4 \pi}\left(g^{2}, g^{\prime 2}\right)$, and $\left(g, g^{\prime}\right)$ denote the weak and hypercharge gauge couplings, respectively. As we have checked, the above gauge interaction contributions agree with Ref. [7]. The Yukawa interactions can induce the $s$-channel annihilations $\chi_{1} \chi_{1} \rightarrow \phi \phi$ and the $t$-channel annihilations $\chi_{1} \chi_{1} \rightarrow \Delta \Delta^{\dagger}$, as well as the related coannihilation channels. In our analysis, we consider the Higgs triplet mass to be around the scale of DM mass (with $M_{\Delta}<2 M_{\chi_{1}}$ ) and the Yukawa couplings to be reasonably small $[f$, $\left.f^{\prime}=\mathcal{O}(0.1-0.01)\right]$. Thus, we find that the (co)annihilation processes $\chi_{1,2} \chi_{1,2} \rightarrow \phi \phi$ are suppressed by the product of the $\Delta \phi \phi$ coupling and $s$-channel propagator factor, which is proportional to $\left(\mu_{\Delta \phi} / M_{\Delta}^{2}\right)^{2} \propto v_{\Delta}^{2} / v_{\phi}^{4}$, where $v_{\Delta}^{2} / v_{\phi}^{2} \ll 1$ [due to Eq. (14)] and $M_{\Delta}$ is chosen significantly away from the resonant production of $\Delta$. For the (co)annihilation processes such as $\chi_{1} \chi_{1} \rightarrow \Delta \Delta^{\dagger}$ and $\chi_{1} \Delta \rightarrow \chi_{1} \Delta$, the effective cross sections will be suppressed by the Yukawa coupling factors $\left(f^{4}, f^{\prime 4}, f^{2} f^{\prime 2}\right)$ for $f, f^{\prime}=\mathcal{O}(0.1-0.01)$. With the above consideration, our model realizes the conventional minimal DM scenario, with gauge interactions dominating the DM annihilation and coannihilation processes as in Eq. (34).

For the current study, the corresponding DM relic density can be expressed as $[18,19]$

$\frac{n_{\chi_{1}}}{s} \simeq \frac{x_{f}}{\langle\sigma v\rangle M_{\chi_{1}} M_{\mathrm{Pl}}}\left(\frac{180}{\pi g_{*}}\right)^{\frac{1}{2}}, \quad x_{f} \simeq \ln \frac{\langle\sigma v\rangle M_{\chi_{1}} M_{\mathrm{Pl}}}{60 \sqrt{g_{*}}}$,

where $n_{\chi_{1}}$ is the DM number density at the freeze-out temperature $T_{f}, s$ is their total entropy, $M_{\mathrm{Pl}} \simeq 1.22 \times$ $10^{19} \mathrm{GeV}$ is the Planck mass, and the ratio $x_{f}=M_{\chi_{1}} / T_{f}$. The quantity $g_{*}$ denotes the effective relativistic d.o.f. in the thermal equilibrium at the freeze-out temperature, and thus $g_{*}=106.75$. For the $\Lambda \mathrm{CDM}$ cosmology, the latest Planck data give $\Omega_{\mathrm{DM}} h^{2}=0.120 \pm 0.001$ [20]. With the generic relation,

$$
\frac{n_{\chi_{1}}}{s}=\frac{0.436 \mathrm{eV} \Omega_{\chi_{1}} h^{2}}{M_{\chi_{1}}} \frac{0.120}{,}
$$

and using Eqs. (34) and (35), we compute the mass of the lightest fermion $\chi_{1}$ as a stable DM particle, 


$$
M_{\chi_{1}} \simeq 1.24 \mathrm{TeV} \text {. }
$$

Because of the small VEV $\left|v_{\Delta}\right| \leq 3.1 \mathrm{GeV}$ and the value of rotation angle $\theta \simeq \frac{\pi}{4}$, the spin-dependent elastic scattering of the DM particle $\chi_{1}$ off the nucleon will be far below the experimental sensitivities. As for the spin-independent elastic scattering, its cross section can be computed at one-loop level and estimated as [7]

$$
\sigma_{\mathrm{SI}} \simeq 3 \times 10^{-46} \mathrm{~cm}^{2},
$$

which is reachable by the future direct detection experiments [16]. Furthermore, the DM particle $\chi_{1}$ and its heavier partner $\chi_{2}$ have a spin-independent inelastic scattering off the nucleon at tree level. If the $\chi_{1}-\chi_{2}$ mass splitting would vanish, the spin-independent inelastic scattering cross section would take the form [21]

$$
\begin{aligned}
\sigma_{0} & =\frac{m_{p}^{2} G_{F}^{2}}{128 \pi}\left[\frac{(A-Z)-\left(1-4 \sin ^{2} \theta_{W}\right) Z}{A}\right]^{2} \\
& =1.16 \times 10^{-40} \mathrm{~cm}^{2}\left[\frac{(A-Z)-\left(1-4 \sin ^{2} \theta_{W}\right) Z}{A}\right]^{2}
\end{aligned}
$$

and would already be excluded by the direct DM searches. In the above equation, $m_{p} \simeq 938 \mathrm{MeV}$ is the proton mass, $G_{F} \simeq 1.16638 \times 10^{-5} \mathrm{GeV}^{-2}$ is the Fermi constant, and $(Z, A)$ denote the (charge, mass) numbers of the target nuclei. On the other hand, our inelastic DM model predicts small but nonzero $\chi_{1}-\chi_{2}$ mass splitting in the range of Eq. (26) or Eq. (27). Hence, the corresponding inelastic DM scattering can escape from the current experimental constraints. Other inelastic DM models have a similar feature regarding the experimental direct detection constraints, as discussed in the literature [22-25].

\section{RADIATIVE TYPE-II NEUTRINO SEESAW}

After the electroweak symmetry breaking, we can generate a Majorana mass term for the left-handed neutrinos via a radiative type-II seesaw, as given by the one-loop diagram in Fig. 1,

$$
\mathcal{L}_{\text {mass }}^{\nu}=-\frac{1}{2} \overline{\nu_{L}^{c}} \mathbf{m}_{\nu} \nu_{L}+\text { H.c. }=\frac{1}{2} \nu^{T} \mathbf{m}_{\nu} \nu+\text { H.c. },
$$

where $\nu_{L}=\left(\begin{array}{l}\nu \\ 0\end{array}\right)$ and $\nu$ is a left-handed Weyl spinor in the $\left(\frac{1}{2}, 0\right)$ representation of the Lorentz group. We note that in Fig. 1 the lepton-number violation $(\Delta L=2)$ arises from the $\sigma$ propagator with the mass insertion $\tilde{M}_{\sigma j}^{2} \propto M_{\sigma j R}^{2}-M_{\sigma j I}^{2}$. The Majorana nature of the neutrino mass generation originates from the vertices $\Delta-\psi_{L}-\psi_{L}$ and $\Delta^{\dagger}-\psi_{L}^{\prime}-\psi_{L}^{\prime}$. From Fig. 1, we compute the one-loop radiative neutrino masses as follows:

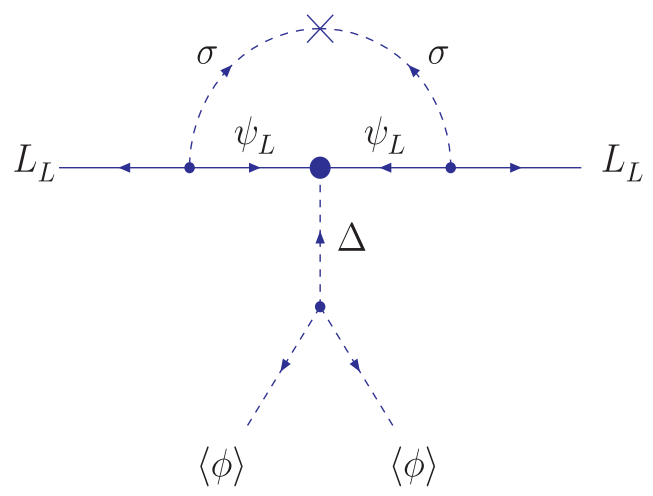

FIG. 1. One-loop diagram for generating the radiative neutrino masses through the type-II seesaw in the present model.

$$
\begin{aligned}
\left(\mathbf{m}_{\nu}\right)_{\alpha \beta}= & \frac{M_{\chi_{1}}}{16 \pi^{2}} \sum_{j} y_{\alpha j} y_{\beta j}\left[\frac{M_{\sigma_{j R}}^{2}}{M_{\sigma_{j R}}^{2}-M_{\chi_{1}}^{2}} \ln \left(\frac{M_{\sigma_{j R}}^{2}}{M_{\chi_{1}}^{2}}\right)\right. \\
& \left.-\frac{M_{\sigma_{j I}}^{2}}{M_{\sigma_{j I}}^{2}-M_{\chi_{1}}^{2}} \ln \left(\frac{M_{\sigma_{j I}}^{2}}{M_{\chi_{1}}^{2}}\right)\right] \\
& -\frac{M_{\chi_{2}}}{16 \pi^{2}} \sum_{j} y_{\alpha j} y_{\beta j}\left[\frac{M_{\sigma_{j R}}^{2}}{M_{\sigma_{j R}}^{2}-M_{\chi_{2}}^{2}} \ln \left(\frac{M_{\sigma_{j R}}^{2}}{M_{\chi_{2}}^{2}}\right)\right. \\
& \left.-\frac{M_{\sigma_{i I}}^{2}}{M_{\sigma_{j I}}^{2}-M_{\chi_{2}}^{2}} \ln \left(\frac{M_{\sigma_{j I}}^{2}}{M_{\chi_{2}}^{2}}\right)\right] .
\end{aligned}
$$

As we will show later, we are interested in the case in which the scalars $\left(\sigma_{j R}, \sigma_{j I}\right)$ can realize the high-scale leptogensis and thus are much heavier than the fermions $\left(\chi_{1}, \chi_{2}\right)$. In this case, we can simplify the above mass formula as

$$
\left(\mathbf{m}_{\nu}\right)_{\alpha \beta} \simeq \frac{\Delta M_{\chi}}{16 \pi^{2}} \sum_{j} y_{\alpha j} y_{\beta j} \ln \left(\frac{M_{\sigma_{j R}}^{2}}{M_{\sigma_{j l}}^{2}}\right)
$$

for $M_{\sigma_{j R}}^{2} \neq M_{\sigma_{j I}}^{2} \gg M_{\chi_{1,2}}^{2}$. Inspecting Eqs. (41) and (42), we see that the neutrino mass $\mathbf{m}_{\nu}$ vanishes if $M_{\sigma_{j R}}=M_{\sigma_{j l}}$. This is expected because the lepton-number violation $(\Delta L=2)$ of the neutrino mass term is generated by the mass insertion of the $\sigma$ field in Fig. 1, $\tilde{M}_{\sigma j}^{2} \propto M_{\sigma j R}^{2}-M_{\sigma j I}^{2}$. Furthermore, Eq. (41) shows that $\mathbf{m}_{\nu}=0$ if $M_{\chi_{1}}=M_{\chi_{2}}$, since the contributions of $\chi_{1}$ and $\chi_{2}$ to the loop diagram of Fig. 1 take the same form but with opposite signs. This is manifest in Eq. (42), in which the neutrino mass is proportional to the mass difference $\Delta M_{\chi}$ and thus the triplet VEV, $\mathbf{m}_{\nu} \propto \Delta M_{\chi} \propto v_{\Delta}$. In addition, we note that for $M_{\sigma_{j R, I}}^{2} \gg$ $M_{\chi 1,2}^{2}$ the mass scale of the loop diagram in Fig. 1 is controlled by the heavy mass of $\sigma_{j}$, so the resultant approximate neutrino-mass formula (42) is controlled by the heavy $\sigma_{j}$ mass and does not depend on the small TeV-scale masses of $\left(\chi_{1}, \chi_{2}\right)$, except the overall 
mass-difference factor $\Delta M_{\chi} \propto v_{\Delta}$ due to the VEV of the external triplet field $\Delta$. This feature is important for our following interpretation of neutrino mass generation via type-II seesaw around Eqs. (43) and (44).

To generate the required size of neutrino masses $m_{\nu}=\mathcal{O}(0.1 \mathrm{eV})$, we set the Yukawa couplings as $y \gtrsim$ $\mathcal{O}\left(10^{-4}\right)$ for $\Delta M_{\chi} \lesssim 17.1 \mathrm{GeV}$, or $y \gtrsim \mathcal{O}\left(10^{-2}\right)$ for $\Delta M_{\chi} \lesssim 550 \mathrm{keV}$, since the logarithm function usually has a value of $\mathcal{O}(1)$. Note that, here, we have a rank-2 neutrino mass matrix $\mathbf{m}_{\nu}$ with two nonzero eigenvalues because the model only contains two scalar singlets $\sigma_{1,2}$ and their Yukawa couplings $y_{\alpha j}$ form a $3 \times 2$ matrix. (This feature is similar to the minimal type-I neutrino seesaw with two right-handed heavy Majorana neutrinos [26].) If the light neutrinos have three nonzero mass eigenvalues, then we can readily extend the present model with three singlet scalars $\sigma_{j}(j=1,2,3)$, which will not affect the main feature of the present model.

The neutrino mass generation (42) may be also understood as a type-II seesaw. Since the scalar singlets $\left(\sigma_{1}, \sigma_{2}\right)$ have masses around the leptogenesis scale (cf. Sec. V) and are extremely heavy, we can expand the exact mass formula (41) to obtain Eq. (42). As we explained below Eq. (42), the loop diagram of Fig. 1 is controlled by the heavy mass of the singlet $\sigma_{j}$, and the resultant approximate formula (42) does not depend on the small masses of the light fields $\left(\chi_{1}, \chi_{2}\right)$ in the loop diagram, except the overall mass-difference factor $\Delta M_{\chi} \propto v_{\Delta}$ due to the VEV of the external triplet field $\Delta$. Thus, it is instructive to view this as integrating out the heavy singlets $\left(\sigma_{1}, \sigma_{2}\right)$, and we obtain the low-energy effective Yukawa interactions between the Higgs triplet $\Delta$ and the SM lepton doublets $L_{L}$,

$$
\mathcal{L}_{\text {eff }}^{Y}=-\frac{1}{2} f_{\text {eff }} \overline{L_{L}^{c}} i \tau_{2} \Delta L_{L}+\text { H.c. }
$$

where $f_{\text {eff }}$ is the effective Yukawa coupling with

$$
\left(f_{\text {eff }}\right)_{\alpha \beta}=\frac{f+f^{\prime}}{16 \pi^{2}} \sum_{j} y_{\alpha j} y_{\beta j} \ln \left(\frac{M_{\sigma_{j R}}^{2}}{M_{\sigma_{j l}}^{2}}\right),
$$

for $M_{\Delta} \ll M_{\sigma_{j R}} \neq M_{\sigma_{j I}}$. We may call the above as a radiative type-II neutrino seesaw since it is realized at one-loop level. For such neutrino mass generation with a fairly small Higgs triplet VEV, $v_{\Delta} \lesssim 0.1 \mathrm{MeV}$, it can be tested at the LHC [17].

For a further remark, we inspect the effective coupling (44) and note that $f_{\text {eff }}$ and the corresponding Majorana neutrino mass would vanish if the mass splitting $M_{\sigma_{j R}}^{2}-$ $M_{\sigma_{j l}}^{2}\left(=2 \tilde{M}_{\sigma j}^{2}\right)$ were equal to zero. This is because the mass splitting between the real and imaginary components of $\sigma_{j}$ arises from the soft lepton number breaking via the mass term of $\tilde{M}_{\sigma j}^{2}$, as we explained below Eq. (42). When the $\sigma_{j}$ mass goes to infinity for any fixed finite massdifference between $\left(\sigma_{j R}, \sigma_{j I}\right)$, we see that the effective coupling (44) also approaches zero, which is consistent with the decoupling theorem.

\section{REALIZING NATURAL HIGH-SCALE LEPTOGENESIS}

In the present model, we can realize a leptogenesis through the decays of the real scalars $\sigma_{i R}$ or $\sigma_{i I}$. The relevant Feynman diagrams are shown in Fig. 2. Thus, we compute the decay widths at tree level,

$$
\begin{aligned}
\Gamma_{\sigma_{i R}} & \equiv \sum_{\alpha}\left[\Gamma\left(\sigma_{i R} \rightarrow L_{L \alpha}+\psi_{L}\right)+\Gamma\left(\sigma_{i R} \rightarrow L_{L \alpha}^{c}+\psi_{L}^{c}\right)\right] \\
& =\frac{1}{8 \pi}\left(y^{\dagger} y\right)_{i i} M_{\sigma_{i R}} \\
\Gamma_{\sigma_{i I}} & \equiv \sum_{\alpha}\left[\Gamma\left(\sigma_{i I} \rightarrow L_{L \alpha}+\psi_{L}\right)+\Gamma\left(\sigma_{i I} \rightarrow L_{L \alpha}^{c}+\psi_{L}^{c}\right)\right] \\
& =\frac{1}{8 \pi}\left(y^{\dagger} y\right)_{i i} M_{\sigma_{i I}} .
\end{aligned}
$$
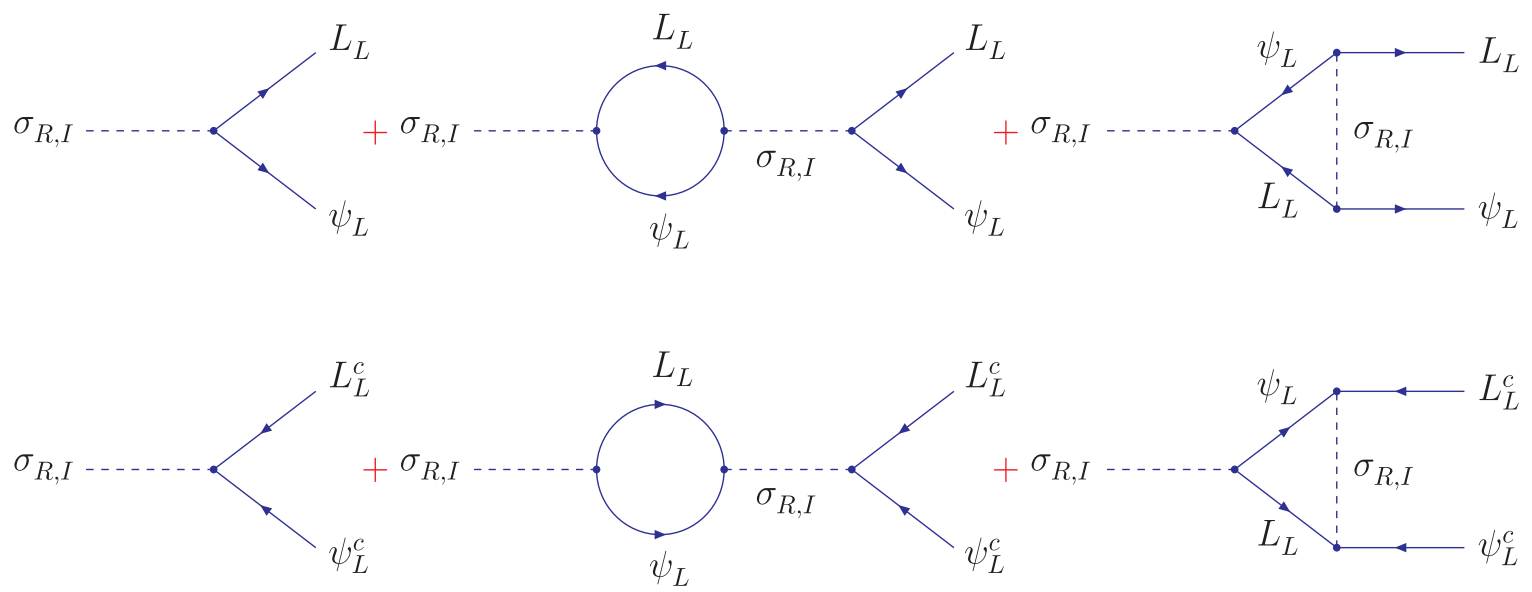

FIG. 2. Decays of the singlet scalars $\sigma_{j}=\frac{1}{\sqrt{2}}\left(\sigma_{R}+i \sigma_{I}\right)$ into the SM lepton doublets $L_{L}$ and the new fermion doublet $\psi_{L}$. 
Then, we evaluate the $C P$ asymmetries at one-loop order,

$$
\begin{aligned}
\varepsilon_{\sigma_{i R}} \equiv & \frac{\sum_{\alpha}\left[\Gamma\left(\sigma_{i R} \rightarrow L_{L \alpha}+\psi_{L}\right)-\Gamma\left(\sigma_{i R} \rightarrow L_{L \alpha}^{c}+\psi_{L}^{c}\right)\right]}{\Gamma_{\sigma_{i R}}} \\
= & \frac{1}{24 \pi} \frac{\operatorname{Im}\left\{\left[\left(y^{\dagger} y\right)_{i j}\right]^{2}\right\}}{\left(y^{\dagger} y\right)_{i i}}\left\{\left[S\left(\frac{M_{\sigma_{j R}}^{2}}{M_{\sigma_{i R}}^{2}}\right)+V\left(\frac{M_{\sigma_{j R}}^{2}}{M_{\sigma_{i R}}^{2}}\right)\right]\right. \\
& \left.-\left[S\left(\frac{M_{\sigma_{j I}}^{2}}{M_{\sigma_{i R}}^{2}}\right)+V\left(\frac{M_{\sigma_{j I}}^{2}}{M_{\sigma_{i R}}^{2}}\right)\right]\right\}, \\
\varepsilon_{\sigma_{i I}} \equiv & \frac{\sum_{\alpha}\left[\Gamma\left(\sigma_{i I} \rightarrow L_{L \alpha}+\psi_{L}\right)-\Gamma\left(\sigma_{i I} \rightarrow L_{L \alpha}^{c}+\psi_{L}^{c}\right)\right]}{\Gamma_{\sigma_{i I}}} \\
= & -\frac{1}{24 \pi} \sum_{j \neq i} \frac{\operatorname{Im}\left\{\left[\left(y^{\dagger} y\right)_{i j}\right]^{2}\right\}}{\left(y^{\dagger} y\right)_{i i}}\left\{\left[S\left(\frac{M_{\sigma_{j R}}^{2}}{M_{\sigma_{i I}}^{2}}\right)+V\left(\frac{M_{\sigma_{j R}}^{2}}{M_{\sigma_{i I}}^{2}}\right)\right]\right. \\
& \left.-\left[S\left(\frac{M_{\sigma_{j I}}^{2}}{M_{\sigma_{i I}}^{2}}\right)+V\left(\frac{M_{\sigma_{j I}}^{2}}{M_{\sigma_{i I}}^{2}}\right)\right]\right\},
\end{aligned}
$$

where $S(x)$ and $V(x)$ are the self-energy and vertex corrections, respectively,

$$
\begin{aligned}
& S(x)=\frac{2}{x-1}, \\
& V(x)=(1+2 x)\left[2+(1+2 x) \ln \frac{x}{1+x}\right] .
\end{aligned}
$$

For an example, we set the masses of the four real scalars $\left(\sigma_{1 R}, \sigma_{1 I}\right)$ and $\left(\sigma_{2 R}, \sigma_{2 I}\right)$ with the following hierarchy:

$$
M_{\sigma_{1 I}}^{2} \ll M_{\sigma_{1 R}}^{2} \ll M_{\sigma_{2 l}}^{2} \ll M_{\sigma_{2 R}}^{2} .
$$

Thus, the final baryon asymmetry should mainly come from the decays of the lightest singlet scalar $\sigma_{1 I}$. In this case, we find that the relevant $C P$ asymmetry becomes

$$
\varepsilon_{\sigma_{1 I}} \simeq \frac{1}{8 \pi} \frac{\operatorname{Im}\left\{\left[\left(y^{\dagger} y\right)_{12}\right]^{2}\right\}}{\left(y^{\dagger} y\right)_{11}} \frac{M_{\sigma_{1 I}}^{2}}{M_{\sigma_{2 I}}^{2}} .
$$

Note that, due to the trilinear scalar vertex $\phi \phi \Delta$ in Eq. (5) and the radiative Yukawa vertex $L_{L} L_{L} \Delta$ in Eq. (43), the Higgs triplet $\Delta$ will mediate some $\Delta L=2$ processes: $L_{L} L_{L} \leftrightarrow \phi \phi, L_{L}^{c} L_{L}^{c} \leftrightarrow \phi^{*} \phi^{*}$, and $L_{L} \phi^{*} \leftrightarrow L_{L}^{c} \phi$. Before the sphalerons (for leptogenesis) stop working, these additional lepton number-violating processes should keep out of the equilibrium and thus do not wash out the produced lepton asymmetry from $\sigma_{1 I}$ decays. This will require

$$
\Gamma_{\Delta L=2}<H(T),
$$

for $T>T_{\text {sph. }}$. Here, the Hubble constant $H(T)$ is given by $H(T)=\left(\frac{8 \pi^{3} g_{*}}{90}\right)^{\frac{1}{2}} \frac{T^{2}}{M_{\mathrm{Pl}}}$, with $M_{\mathrm{Pl}} \simeq 1.22 \times 10^{19} \mathrm{GeV}$ being the
Planck mass and $g_{*}=\mathcal{O}(100)$ denoting the relativistic d.o.f. during the leptogenesis epoch. As for the rate of lepton number-violating interactions, $\Gamma_{\Delta L=2}$, we can estimate

$$
\begin{aligned}
\Gamma_{\Delta L=2} & \sim \frac{\operatorname{Tr}\left(f_{\text {eff }}^{\dagger} f_{\text {eff }}\right)\left|\mu_{\Delta \phi}\right|^{2}}{T} \\
& =\frac{4 M_{\Delta}^{4} \operatorname{Tr}\left(m_{\nu}^{\dagger} m_{\nu}\right)}{v_{\phi}^{4} T}, \quad\left(\text { for } T>M_{\Delta}\right), \\
\Gamma_{\Delta L=2} & \sim \frac{\operatorname{Tr}\left(f_{\text {eff }}^{\dagger} f_{\text {eff }}\right)\left|\mu_{\Delta \phi}\right|^{2} T^{3}}{M_{\Delta}^{4}} \\
& =\frac{4 \operatorname{Tr}\left(m_{\nu}^{\dagger} m_{\nu}\right) T^{3}}{v_{\phi}^{4}}, \quad\left(\text { for } T<M_{\Delta}\right) .
\end{aligned}
$$

For a numerical demonstration, we define

$$
K=\left.\frac{\Gamma_{\sigma_{1 I}}}{2 H(T)}\right|_{T=M_{\sigma_{1 I}}},
$$

where $H(T)$ is the Hubble constant given by Eq. (33). In the weak washout region, the final baryon asymmetry can be described as [18]

$$
\eta_{B}=\frac{n_{B}}{s} \simeq-\frac{28}{79} \times \frac{\varepsilon_{\sigma_{1 I}}}{g_{*}},
$$

for $K \ll 1$. Here, $n_{B}$ and $s$ denote the baryon number density and the entropy density, respectively, while the factor $-\frac{28}{79}$ is the sphaleron lepton-to-baryon coefficient. In the present model, we have $g_{*}=119.75$ accounting for the SM fields plus one Higgs triplet $(\Delta)$ and two fermion doublets $\left(\psi_{L}\right.$ and $\left.\psi_{L}^{\prime}\right)$. The latest Planck observation gives [20],

$$
\eta_{B}=(6.12 \pm 0.03) \times 10^{-10} .
$$

For illustration, it is useful to define a simple effective coupling $\bar{y}_{\mathrm{TL}}$ to characterize the size of the relevant Yukawa couplings for the $L_{L}-\psi_{L^{-}} \sigma$ vertex (especially, its order of magnitude), without invoking the detailed structure of Yukawa matrix,

$$
\bar{y}_{\mathrm{TL}}^{2} \equiv-\frac{\operatorname{Im}\left\{\left[\left(y^{\dagger} y\right)_{12}\right]^{2}\right\}}{\left(y^{\dagger} y\right)_{11}} .
$$

Thus, we have

$$
\bar{y}_{\mathrm{TL}} \simeq \sqrt{\frac{158 g_{*} \eta_{B}}{7}}\left(\frac{M_{\sigma_{2 I}}}{M_{\sigma_{11}}}\right) .
$$

With this, we impose the baryon asymmetry data (54) and estimate the allowed range of the effective Yukawa 


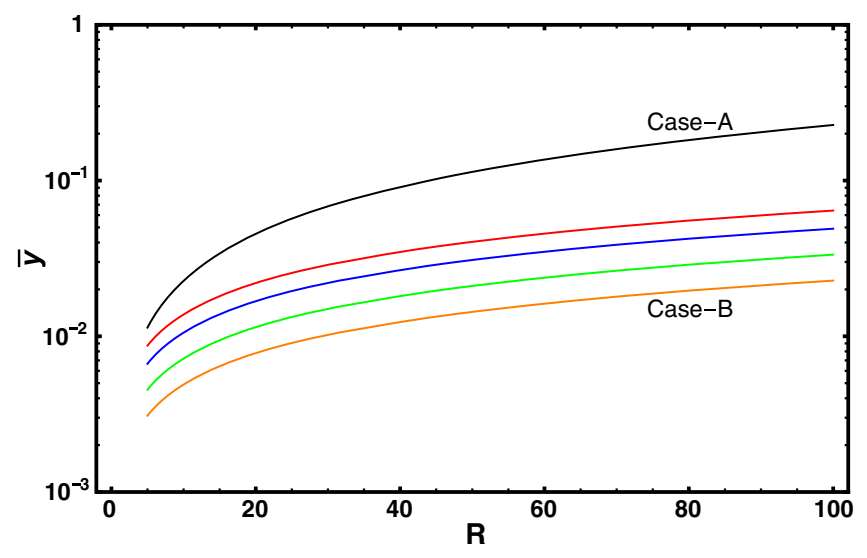

FIG. 3. The characteristic size of effective Yukawa coupling $\bar{y}$ as a function of the scalar mass ratio $R=M_{\sigma_{2 I}} / M_{\sigma_{1 I}}$ under the constraint of baryon asymmetry (54), where $\bar{y}=\bar{y}_{\mathrm{TL}}$ is defined in Eq. (55) for case A and $\bar{y}=\bar{y}_{\mathrm{nTL}}$ in Eq. (61) for case B. In case $B$, the (yellow, green, blue, red) curves from the bottom to top correspond to the lowest singlet scalar mass $M_{\sigma_{1 I}}=\left(10^{13}, 10^{14}, 10^{15}, 5 \times 10^{15}\right) \mathrm{GeV}$.

coupling $\bar{y}_{\mathrm{TL}}$ as a function of the scalar mass ratio $R=M_{\sigma_{2 l}} / M_{\sigma_{1 I}}$. We plot this as the black curve (called case A) in Fig. 3 for the range of $5 \leq R \leq 10^{2}$. We input the experimental central value of Eq. (54). We also vary the value of $\eta_{B}$ within $\pm 3 \sigma$ range but find no visible effect in Fig. 3. From Fig. 3, we see that the typical size of Yukawa couplings $\left(\bar{y}_{\mathrm{TL}}\right)$ can be naturally around $\mathcal{O}\left(10^{-1}-10^{-2}\right)$.

For an explicit numerical sample, we can choose the sample inputs,

$$
\begin{aligned}
& M_{\sigma_{1 I}}=10^{-1} M_{\sigma_{2 I}}=1.5 \times 10^{13} \mathrm{GeV}, \\
& M_{\sigma_{1 R}}=10^{-1} M_{\sigma_{2 R}}=7.5 \times 10^{13} \mathrm{GeV}, \\
& \Delta M_{\chi}=550 \mathrm{keV}, \quad \bar{y}_{\mathrm{TL}}=2.27 \times 10^{-2} .
\end{aligned}
$$

Thus, we have

$$
\varepsilon_{\sigma_{1 I}} \simeq 2.1 \times 10^{-7}, \quad \eta_{B} \simeq 6.1 \times 10^{-10},
$$

where the produced baryon asymmetry $\eta_{B}$ is consistent with the recent Planck observation [20] in Eq. (54). We can also estimate $K=\mathcal{O}(0.1)$.

Then, we take another approach and realize a nonthermal leptogenesis. For this, we use the imaginary scalar component $\sigma_{1 I}$ to play the role of an inflaton [27]. In this case, a final baryon asymmetry can be induced [18],

$$
\eta_{B}=-\frac{28}{79} \frac{T_{\mathrm{RH}}}{M_{\sigma_{1 I}}} \varepsilon_{\sigma_{1 I}}
$$

with $T_{\mathrm{RH}}$ being the reheating temperature [18],

$$
\begin{aligned}
T_{\mathrm{RH}} & \equiv T\left(t=\Gamma_{\sigma_{1 I}}^{-1}\right) \\
& =\left(\frac{90}{8 \pi^{3} g_{*}}\right)^{\frac{1}{4}} \sqrt{\frac{\left(y^{\dagger} y\right)_{11} M_{\mathrm{Pl}} M_{\sigma_{1 I}}}{16 \pi}} .
\end{aligned}
$$

For illustration of this model, we may define a simple effective coupling $\bar{y}_{\mathrm{nTL}}$ to characterize the size of the relevant Yukawa couplings for the $L_{L}-\psi_{L}-\sigma$ vertex,

$$
\bar{y}_{\mathrm{nTL}}^{3} \equiv-\frac{\operatorname{Im}\left\{\left[\left(y^{\dagger} y\right)_{12}\right]^{2}\right\}}{\sqrt{\left(y^{\dagger} y\right)_{11}}} .
$$

Thus, we can express $\bar{y}_{\mathrm{nTL}}$ as follows:

$\bar{y}_{\mathrm{nTL}}=\left[\eta_{B} \frac{158 \pi}{7}\left(\frac{8 \pi^{3} g_{*}}{90}\right)^{\frac{1}{4}}\left(\frac{16 \pi M_{\sigma_{1 I}}}{M_{\mathrm{Pl}}}\right)^{\frac{1}{2}}\right]^{\frac{1}{3}}\left(\frac{M_{\sigma_{2 I}}}{M_{\sigma_{1 I}}}\right)^{\frac{2}{3}}$.

Next, imposing the baryon asymmetry data (54), we can estimate the allowed range of the effective Yukawa coupling $\bar{y}_{\mathrm{nTL}}$ as a function of the scalar mass ratio $R=M_{\sigma_{2 l}} / M_{\sigma_{1 I}}$. In Fig. 3, we plot this as the lower set of curves in (yellow, green, blue, red) colors (called case B) from the bottom to top, corresponding to $M_{\sigma_{1 I}}=$ $\left(10^{13}, 10^{14}, 10^{15}, 5 \times 10^{15}\right) \mathrm{GeV}$, for $5 \leq R \leq 10^{2}$.

For an explicit numerical sample of this model, we make the parameter choice

$$
\begin{aligned}
& M_{\sigma_{1 I}}=20^{-1} M_{\sigma_{2 l}}=4.5 \times 10^{13} \mathrm{GeV}, \\
& M_{\sigma_{1 R}}=20^{-1} M_{\sigma_{2 R}}=2.3 \times 10^{14} \mathrm{GeV}, \\
& \Delta M_{\chi}=550 \mathrm{keV}, \quad \bar{y}_{\mathrm{nTL}}=10^{-2},
\end{aligned}
$$

and derive

$$
\eta_{B} \simeq 6.1 \times 10^{-10},
$$

which is consistent with the Planck observation (54) [20]. For illustration, we further choose a typical input $\left(y^{\dagger} y\right)_{11}^{1 / 2}=$ $10^{-2}$ and estimate

$$
\varepsilon_{\sigma_{1 I}} \simeq 10^{-9}, \quad T_{\mathrm{RH}} \simeq 7.6 \times 10^{12} \mathrm{GeV} .
$$

In the above samples, we choose $\Delta M_{\chi}=550 \mathrm{keV}$ and the effective Yukawa coupling $\bar{y}_{\mathrm{TL}}, \bar{y}_{\mathrm{nTL}}=\mathcal{O}\left(10^{-2}\right)$. Thus, from the radiative mass formula (42) of light neutrinos, we see that the light neutrino mass scale of $m_{\nu}=\mathcal{O}(0.1 \mathrm{eV})$ can be realized, which is consistent with the oscillation data [3] and cosmological constraints [20]. Also, for the above numerical samples, we have checked that the condition (50) is satisfied for the parameter choice (57) and (63). In particular, Eq. (51a) will match the condition (50) during the leptogenesis epoch $T \gg M_{\Delta}$ and the lowtemperature period $T \sim M_{\Delta}$. When the temperature falls to $T<M_{\Delta}$, the rate will become as Eq. (51b) and is reduced 
by a factor $T^{4} / M_{\Delta}^{4}<1$. This means that Eq. (51b) also matches the condition (50).

\section{CONCLUSIONS}

Understanding the origins of the neutrino masses, the baryon asymmetry, and the dark matter altogether poses an important challenge to particle physics today. In the conventional seesaw framework, the neutrino mass generation and the leptogenesis for baryon asymmetry are tied to the same high-energy scale. This means that a low-scale neutrino mass generation could not be consistent with a high-scale leptogenesis. In the present work, we demonstrated an attractive new possibility that a radiative neutrino mass generation can be achieved at the $\mathrm{TeV}$ scale, while a thermal or inflationary leptogenesis naturally happens at the high scale. Furthermore, our model realizes a viable minimal inelastic dark matter at the $\mathrm{TeV}$ scale, where the mass splitting between the DM particle and its heavier partner can be naturally generated by the interactions related to the neutrino mass generation.

In Sec. II, we presented the model construction, which extends the standard model with two gauge-singlet scalars $\left(\sigma_{1}, \sigma_{2}\right)$, a vectorlike isodoublet fermion $\left(\psi_{L}, \psi_{L}^{\prime c}\right)$ and one isotriplet Higgs $\Delta$. This model holds a softly broken lepton number and an exactly conserved $\mathbb{Z}_{2}$ discrete symmetry. Then, in Sec. III, we showed that the lighter Majorana fermion $\chi_{1}$ can serve as a stable DM candidate and provide the observed relic density in the present Universe with its mass $M_{\chi_{1}} \simeq 1.24 \mathrm{TeV}$. This fermionic DM $\chi_{1}$ can be searched by the current direct/indirect DM detection experiments [16] and by the ongoing LHC experiments as well as the future high-energy $p p$ colliders [28]. In Sec. IV, we demonstrated how our model can naturally realize the minimal type-II seesaw and radiatively generate the light neutrino masses $m_{\nu}=\mathcal{O}(0.1 \mathrm{eV})$ at the TeV scale [cf. Fig. 1 and Eq. (42)]. Finally, in Sec. V, we studied the realization of a natural thermal or inflationary leptogenesis through decays of the lightest singlet scalar $\sigma_{1 I}$ at a high scale around $\mathcal{O}\left(10^{13} \mathrm{GeV}\right)$.

\section{ACKNOWLEDGMENTS}

We thank Alessandro Strumia for discussing the minimal dark matter models. P. H. G. was supported by the National Natural Science Foundation of China under Grant No. 11675100 and the Recruitment Program for Young Professionals under Grant No. 15Z127060004. H. J. H. was supported in part by the National NSF of China (under Grants No. 11675086 and No. 11835005) and the National Key R \& D Project of China (under Grant No. 2017YFA0402204); he was also supported in part by the Shanghai Laboratory for Particle Physics and Cosmology (under Grant No. 11DZ2260700) and the Office of Science and Technology, Shanghai Municipal Government (under Grant No. 16DZ2260200).
[1] P. Minkowski, Phys. Lett. B 67, 421 (1977); T. Yanagida, in Proceedings of the Workshop on Unified Theory and the Baryon Number of the Universe, edited by O. Sawada and A. Sugamoto (KEK, Tsukuba, 1979), p. 95; M. Gell-Mann, P. Ramond, and R. Slansky, in Supergravity, edited by F. van Nieuwenhuizen and D. Freedman (North Holland, Amsterdam, 1979), p. 315; S. L. Glashow, in Quarks and Leptons, edited by M. Lévy et al. (Plenum, New York, 1980), p. 707; R. N. Mohapatra and G. Senjanović, Phys. Rev. Lett. 44, 912 (1980).

[2] J. Schechter and J. W. F. Valle, Phys. Rev. D 22, 2227 (1980); M. Magg and C. Wetterich, Phys. Lett. B 94, 61 (1980); T. P. Cheng and L.F. Li, Phys. Rev. D 22, 2860 (1980); G. Lazarides, Q. Shafi, and C. Wetterich, Nucl. Phys. B181, 287 (1981); R. N. Mohapatra and G. Senjanović, Phys. Rev. D 23, 165 (1981).

[3] K. A. Olive et al. (Particle Data Group), Chin. Phys. C 40, 100001 (2016).

[4] M. Fukugita and T. Yanagida, Phys. Lett. B 174, 45 (1986).

[5] P. Langacker, R. D. Peccei, and T. Yanagida, Mod. Phys. Lett. A 01, 541 (1986); M. A. Luty, Phys. Rev. D 45, 455 (1992); R. N. Mohapatra and X. Zhang, Phys. Rev. D 46, 5331 (1992).
[6] For a review, W. Buchmuller, R. D. Peccei, and T. Yanagida, Annu. Rev. Nucl. Part. Sci. 55, 311 (2005), and references therein.

[7] M. Cirelli, N. Fornengo, and A. Strumia, Nucl. Phys. B753, 178 (2006).

[8] M. Cirelli, A. Strumia, and M. Tamburini, Nucl. Phys. B787, 152 (2007).

[9] M. Cirelli, T. Hambye, P. Panci, F. Sala, and M. Taoso, J. Cosmol. Astropart. Phys. 10 (2015) 026.

[10] L. M. Krauss, S. Nasri, and M. Trodden, Phys. Rev. D 67, 085002 (2003).

[11] E. Ma, Phys. Rev. D 73, 077301 (2006); J. Kubo, E. Ma, and D. Suematsu, Phys. Lett. B 642, 18 (2006); E. Ma and D. Suematsu, Mod. Phys. Lett. A 24, 583 (2009); E. Ma, Phys. Rev. Lett. 115, 011801 (2015); S. Y. Guo, Z. L. Han, and Y. Liao, Phys. Rev. D 94, 115014 (2016).

[12] P. H. Gu, H. J. He, U. Sarkar, and X. Zhang, Phys. Rev. D 80, 053004 (2009).

[13] W. B. Lu and P. H. Gu, J. Cosmol. Astropart. Phys. 05 (2016) 040; Nucl. Phys. B924, 279 (2017).

[14] For a recent review, Y. Cai, J. Herrero-Garcia, M. A. Schmidt, A. Vicente, and R. R. Volkas, Front. Phys. 5, 63 (2017), and references therein. 
[15] P. H. Gu, J. High Energy Phys. 04 (2017) 159; P. H. Gu and U. Sarkar, Phys. Rev. D 96, 031703 (2017); H. Zhou and P. H. Gu, Nucl. Phys. B927, 184 (2018).

[16] For recent reviews, J. Liu, X. Chen, and X. Ji, Nat. Phys. 13, 212 (2017); D. Bauer et al., Phys. Dark Universe 7-8, 16 (2015); and references therein.

[17] For example, P. F. Perez, T. Han, G. Huang, T. Li, and K. Wang, Phys. Rev. D 78, 015018 (2008); D. K. Ghosh, N. Ghosh, I. Saha, and A. Shaw, Phys. Rev. D 97, 115022 (2018); Y. Cai, T. Han, T. Li, and R. Ruiz, Front. Phys. 6, 40 (2018); and references therein.

[18] E. W. Kolb and M. S. Turner, The Early Universe (AddisonWesley, Reading, MA, 1990).

[19] M. Srednicki, R. Watkins, and K. A. Olive, Nucl. Phys. B310, 693 (1988); P. Gondolo and G. Gelmini, Nucl. Phys. B360, 145 (1991).

[20] N. Aghanim et al. (Planck Collaboration), arXiv:1807.06209.

[21] G. Jungman, M. Kamionkowski, and K. Griest, Phys. Rep. 267, 195 (1996).

[22] D. Tucker-Smith and N. Weiner, Phys. Rev. D 64, 043502 (2001).

[23] C. Arina and N. Sahu, Nucl. Phys. B854, 666 (2012).

[24] C. Arina, R. N. Mohapatra, and N. Sahu, Phys. Lett. B 720, 130 (2013).

[25] J. March-Russell, C. McCabe, and M. McCullough, J. High Energy Phys. 05 (2009) 071; Y. Cui, D. E. Morrissey, D. Poland, and L. Randall, J. High Energy Phys. 05 (2009) 076; S. Chang, G. D. Kribs, D. Tucker-Smith, and N. Weiner, Phys. Rev. D 79, 043513 (2009); C. Arina, F. S. Ling, and M. H. G. Tytgat, J. Cosmol. Astropart. Phys. 10 (2009) 018; K. Schmidt-Hoberg and M. W. Winkler, J. Cosmol. Astropart. Phys. 09 (2009) 010; J. Shu, P. Yin, and S. Zhu, Phys. Rev. D 81, 123519 (2010); D. S. M. Alves, S. R. Behbahani, P. Schuster, and J. G. Wacker,
J. High Energy Phys. 06 (2010) 113; D. S. M. Alves, M. Lisanti, and J. G. Wacker, Phys. Rev. D 82, 031901 (2010); S. Chang, R. F. Lang, and N. Weiner, Phys. Rev. Lett. 106, 011301 (2011); S. Chang, N. Weiner, and I. Yavin, Phys. Rev. D 82, 125011 (2010); H. An, P. S. B. Dev, Y. Cai, and R. N. Mohapatra, Phys. Rev. Lett. 108, 081806 (2012); Y. Bai and T. M. P. Tait, Phys. Lett. B 710, 335 (2012); S. Patra and S. Rao, arXiv:1112.3454; S. Miao, C. L. Shan, and Y. F. Zhou, Int. J. Mod. Phys. A 29, 1450014 (2014); N. Bozorgnia, J. Herrero-Garcia, T. Schwetz, and J. Zupan, J. Cosmol. Astropart. Phys. 07 (2013) 049; L. Baudis, G. Kessler, P. Klos, R. F. Lang, J. Menéndez, S. Reichard, and A. Schwenk, Phys. Rev. D 88, 115014 (2013); S. Scopel and K. H. Yoon, J. Cosmol. Astropart. Phys. 08 (2014) 060; G. Barello, S. Chang, and C. A. Newby, Phys. Rev. D 90, 094027 (2014); E. Izaguirre, G. Krnjaic, and B. Shuve, Phys. Rev. D 93, 063523 (2016); C. McCabe, J. Cosmol. Astropart. Phys. 05 (2016) 033; S. Scopel and K. H. Yoon, J. Cosmol. Astropart. Phys. 02 (2016) 050; M. Blennow, S. Clementz, and J. Herrero-Garcia, J. Cosmol. Astropart. Phys. 04 (2016) 004.

[26] For example, P. H. Frampton, S. L. Glashow, and T. Yanagida, Phys. Lett. B 548, 119 (2002); M. Raidal and A. Strumia, Phys. Lett. B 553, 72 (2003); V. Barger, D. A. Dicus, H. J. He, and T. Li, Phys. Lett. B 583, 173 (2004); S. F. Ge, H. J. He, and F. R. Yin, J. Cosmol. Astropart. Phys. 05 (2010) 017; H. J. He and F. R. Yin, Phys. Rev. D 84, 033009 (2011); W. L. Guo, Z. Z. Xing, and S. Zhou, Int. J. Mod. Phys. E 16, 1 (2007); and references therein.

[27] R. Kallosh, A. Linde, and A. Westphal, Phys. Rev. D 90, 023534 (2014).

[28] M. Bicer et al. (FCC Collaboration), J. High Energy Phys. 01 (2014) 164; CEPC/SPPC Collaboration, http://cepc.ihep .ac.cn. 